\title{
Effect of 8-week Nordic walking training on nondominant hand grip and shoulder strength in middle-aged women
}

\author{
Dong-Jick Kwak, Kyung-Tae Kim, Gyu-Min Kang, Yun-Jin Park, Hae-Lim Lee, Myung-Ki Kim* \\ School of Global Sport Studies, Korea University, Sejong, Korea
}

Nordic walking (NW) is a full body training that can be performed with special pole, and has been recognized as an effective out-door activity which can improve the strength of upper extremity. Most previous studies mainly analyzed the effect of NW on the strength of dominant (DN) hand-grip strength, however, a clear scientific examination is needed whether nondominant (NDN) extremity can also be ameliorated by the NW. Therefore, the aim of our study is to investigate the effectiveness of 8 weeks NW training on the NDN handgrip and shoulder strength in healthy middle-aged women. 29 subjects were randomly divided into three groups: Nordic walking group (NWG, $n=10$ ), brisk walking group (BWG, $n=11$ ), and control group (CG, $n=8)$. Handgrip and shoulder strength were measured by the hand dynamometer and isokinetic machine. NW training was performed 3 times a week, and had 10-min warm-up, 40-min main program consisted of ALFA (Attention, Long arms, Flat poles, Adaptes) technique and Turbo walking, and 10-min cool-down. In handgrip strength of DN hand and the shoulder flexor strength in the both sides, NWG and BWG showed significantly higher value compared to CG. In the NDN hand, NWG also showed significantly higher strength compared to $\mathrm{CG}$, however, there was no significant difference in the handgrip strength/weight among groups. In NDN shoulder extension, significantly higher improvement was found in NWG and BWG compared to that of CG. In conclusion, our study showed that NW can improve the strength of upper extremity in subjects, and can be better approach to improve the NDN handgrip strength than brisk walking method could.

Keywords: Nordic walking, Hand grip strength, Shoulder strength, Middle-aged women

\section{INTRODUCTION}

There has been well-recognized notion that the muscular strength of dominant (DN) upper extremity is greater than that of nondominant (NDN) side, especially in the sedentary population (Bagesteiro and Sainburg, 2002; Goble and Brown, 2008). One study reported that DN hand showed $10 \%$ greater handgrip strength (HS) than NDN did, and defined this phenomenon as a '10\% rule' (Petersen et al., 1989). The difference in HS between both hands was observed by approximately $10 \%$ in the adult men and women subjects, however, '10\% rule' was only applied to right-handed person (12.72\%) while the left-handed subjects showed only $-0.08 \%$ (Petersen et al., 1989). In the case of factory workers frequently performing bimanual tasks (Jarjour et al., 1997), the aforementioned rule may not be applied either since the effectiveness of kinesiological repetitive movement may counterbalance the discrepancy of muscular strength in our body. As the sedentary population may have less chance to actively use both hands in daily life, HS difference between DN and NDN is more likely to occur compared to the other population whose occupational characteristics requires the bimanual-basis activity. In this sense, repetitive kinesiological movement of Nordic walking (NW) using the both DN and NDN will provide a beneficial effect that balances the muscular strength in hand-grip or shoulder.

Although the resistance training can also be an effective physical intervention to increase the muscular strength in upper extremity (Abe et al., 2000) or balance the strength between DN and NDN area, the approachability of NW may take precedence over any other types of activity in terms of time and cost-wise aspects considering NW can be performed in the safe open field.
${ }^{*}$ Corresponding author: Myung-Ki Kim (iD https://orcid.org/0000-0002-5821-9203 School of Global Sport Studies, Korea University, Sejong Campus,

2511 Sejong-ro, Jochiwon-eup, Sejong 30019, Korea

E-mail: kmk1905@korea.ac.kr

Received: April 26, 2019 / Accepted: May 29, 2019
This is an Open Access article distributed under the terms of the Creative Commons Attribution Non-Commercial License (http://creativecommons.org/licenses/by-nc/4.0/) which permits unrestricted non-commercial use, distribution, and reproduction in any medium, provided the original work is properly cited. 
Moreover, as NW is accompanied with the walking pole that can potentially create the natural weight and produce the repulsive force from the ground, its movement characteristics may provide the repetitive light-weight training, which can also prevent the muscular or joint injuries known to be frequently caused by resistance training. Previous studies also reported that NW has a beneficial effect on functional mobility (Virág et al., 2015), lower and upper extremity muscle strength (Church et al., 2002; Song et al., 2013), bone density (Ossowski et al., 2016), aerobic capacity and cardiorespiratory fitness (Figueiredo et al., 2013; Takeshima et al., 2013), however, the effect of NW on the muscular strength of the NDN upper extremity was not clearly verified.

Therefore, the aim of our study is to investigate whether the regular-basis NW training in healthy middle-aged women can effectively balance the difference of muscular difference between $\mathrm{DN}$ and NDN, resulting in the improvement of NDN HS and shoulder strength.

\section{MATERIALS AND METHODS}

\section{Subjects}

Twenty-nine female subjects aged between 44-60 years were recruited from the online social group, 'Mom's café' and a resident in Sejong city, South Korea. Subjects were randomly assigned to three groups, Nordic walking group (NWG, $\mathrm{n}=10$ ), brisk walking group (BWG, $n=11$ ), and control group (CG, $n=8)$ (Table 1). Each

Table 1. Physical characteristics of the subjects $(n=29)$

\begin{tabular}{lccc}
\hline Characteristics & NWG $(\mathrm{n}=10)$ & $\mathrm{BWG}(\mathrm{n}=11)$ & $\mathrm{CG}(\mathrm{n}=8)$ \\
\hline Age $(\mathrm{yr})$ & $57.10 \pm 3.11$ & $53.91 \pm 5.09$ & $53.63 \pm 3.99$ \\
Right hand dominance & $8(80)$ & $9(81.82)$ & $6(75)$ \\
Left hand dominance & $2(20)$ & $2(18.18)$ & $2(25)$ \\
Height $(\mathrm{cm})$ & $158.20 \pm 4.39$ & $160.27 \pm 5.42$ & $157.25 \pm 3.62$ \\
Weight $(\mathrm{kg})$ & $61.01 \pm 9.23$ & $66.37 \pm 7.98$ & $60.24 \pm 9.27$ \\
Body mass index $\left(\mathrm{kg} / \mathrm{m}^{2}\right)$ & $24.46 \pm 4.18$ & $25.85 \pm 2.89$ & $24.28 \pm 3.03$ \\
Hand grip strength $(\mathrm{kg})$ & & & \\
$\quad$ Dominance & $25.63 \pm 3.64$ & $26.86 \pm 3.46$ & $23.75 \pm 2.54$ \\
$\quad$ Nondominance & $23.91 \pm 2.62$ & $24.81 \pm 3.32$ & $22.15 \pm 3.04$ \\
Shoulder Flexion Strength $(\mathrm{Nm})$ & & \\
$\quad$ Dominance & $26.86 \pm 3.97$ & $33.35 \pm 9.01$ & $29.03 \pm 5.80$ \\
$\quad$ Nondominance & $25.46 \pm 4.25$ & $30.34 \pm 6.80$ & $28.13 \pm 5.88$ \\
Shoulder extension strength $(\mathrm{Nm})$ & & \\
$\quad$ Dominance & $34.53 \pm 3.64$ & $34.51 \pm 10.04$ & $30.05 \pm 4.43$ \\
Nondominance & $32.13 \pm 3.76$ & $30.45 \pm 10.99$ & $28.18 \pm 5.10$ \\
\hline
\end{tabular}

Values are present as mean \pm standard deviation or number $(\%)$.

NWG, Nordic walking group; BWG, brisk walking group; CG, control group. group had two subjects using the left hand with dominant side. Experimental participant candidates had no prescribed medication within 6 months, history of musculoskeletal, cardiovascular, or metabolic diseases, and regular exercise experience within 6 months.

\section{HS testing}

The measurement of HS was performed by using a JAMAR hand dynamometer (BK-7498, Fred Sammons Inc., Brookfield, IL, USA). Each subject was seated with the straight posture, and bent the elbow by $90^{\circ}$ with wrist maintained in neutral position. HS was measured three times in the DN and NDN hand with 1-min rest interval between the measurement, and the mean value was obtained for the data analysis. During the measurement, the tester constantly encouraged participants to exert their full effort. The relative HS value was calculated to divide the subject's HS $(\mathrm{kg})$ by each subject's body weight $(\mathrm{BW}, \mathrm{kg}), \mathrm{HS} / \mathrm{BW}$.

\section{Shoulder strength test}

Shoulder strength was measured by using isokinetic machine (Biodex Medical Systems Inc., Shirley, NY, USA). Subjects were seated in the examination chair, and range of motion (ROM) was adjusted using a spring-loaded handle and shoulder attachment apparatus so that the target muscle could be accurately measured. The alignment of the rotational axis of dynamometer was adjusted based on each subject's acromion, and ROM of the shoulder was set between $0^{\circ}$ and $180^{\circ}$. The isokinetic shoulder flexion and extension through concentric contraction were performed 5 times each at $60^{\circ} / \mathrm{sec}$, and the test was administered to both $\mathrm{DN}$ and NDN shoulder.

\section{NW and BW training program}

NW training program was conducted 3 days a week for 8 weeks, and 1-day training session had 60-min program consisted of 10min warm-up, 40-min main exercise, and 10-min cool down. Exercise intensity was monitored via rating of perceived exertion during the 40-min main exercise. BWG was asked to perform the normal stride walking until 4 weeks, and wide stride walking was conducted until 8-week exercise program. For the NWG, ALFA (Attention, Long arms, Flat poles, Adaptes) technique was used until 4 weeks, and they were asked to perform the Turbo walking intervention until 8-week session.

\section{Data analysis}

IBM SPSS Statistics ver. 21.0 (IBM Co., Armonk, NY, USA) was used to obtain the mean \pm standard deviation for the data 
analysis. The one-way analysis of variance was used to verify the homogeneity of the variance. The comparison between the three groups were performed using A two-way repeat measure analysis. When the statistical significance in the data was found, Duncan analysis as a post hoc test was conducted. All statistical significance level was set at $P<0.05$.

\section{RESULTS}

\section{Comparison of HS among groups after 8-weeks intervention}

HS in DN and NDN area was significantly affected by main effect (Group) $(P<0.05)$, and the significant interaction effect
(Group $\times$ Time) $(P<0.01)$ was found in DN and NDN (Table 2). After 8 -weektraining intervention, both NWG and BWG showed a significantly improved HS $(P<0.05)$ compared to CG in DN side, however, it is found that only NWG showed a meaningful improvement $(P<0.01)$ in NDN side compared to CG.

\section{Comparison of relative HS among groups after 8-week intervention}

Relative HS was not affected by main effects (Group and Time) $(P<0.05)$, however, significant interaction effect (Group $\times$ Time) was found in both DN $(P<0.01)$ and NDN $(P<0.001)$ hand. After 8-week NW training, NWG showed significantly improved relative HS in $\mathrm{DN}(P<0.05)$ and NDN hand $(P<0.01)$ (Table 2$)$.

Table 2. Comparison of hand grip strength and shoulder strength among the three groups

\begin{tabular}{|c|c|c|c|c|c|c|c|}
\hline \multirow{2}{*}{ Strength } & \multirow{2}{*}{ Group } & \multirow{2}{*}{ Pre } & \multirow{2}{*}{ Post } & \multirow{2}{*}{$T$} & \multicolumn{3}{|c|}{$F$} \\
\hline & & & & & Group & Time & $\mathrm{G} \times \mathrm{T}$ \\
\hline \multicolumn{8}{|c|}{ Hand grip strength (kg) } \\
\hline \multirow[t]{3}{*}{ Dominance } & NWG & $25.63 \pm 3.64$ & $27.78 \pm 3.06$ & $-2.989^{*}$ & $3.814^{*}$ & 1.405 & $9.208^{* *}$ \\
\hline & BWG & $26.86 \pm 3.46$ & $25.31 \pm 4.68$ & $2.493^{*}$ & & & $a, b>c$ \\
\hline & CG & $23.75 \pm 2.54$ & $21.56 \pm 2.54$ & 2.129 & & & \\
\hline \multirow[t]{3}{*}{ Nondominance } & NWG & $23.91 \pm 2.62$ & $27.46 \pm 3.04$ & $-4.636^{* *}$ & $6.549 * *$ & 0.384 & $14.810^{* * *}$ \\
\hline & BWG & $24.81 \pm 3.32$ & $24.35 \pm 2.87$ & 1.408 & & & $a>c$ \\
\hline & CG & $22.15 \pm 3.04$ & $19.88 \pm 3.40$ & 1.902 & & & \\
\hline \multicolumn{8}{|c|}{ Hand grip strength/weight } \\
\hline \multirow[t]{3}{*}{ Dominance } & NWG & $0.43 \pm 0.10$ & $0.47 \pm 0.08$ & $-3.008^{*}$ & 2.076 & 0.479 & $8.279^{* *}$ \\
\hline & BWG & $0.41 \pm 0.05$ & $0.39 \pm 0.07$ & 1.429 & & & \\
\hline & CG & $0.41 \pm 0.09$ & $0.37 \pm 0.07$ & 2.168 & & & \\
\hline \multirow[t]{3}{*}{ Nondominance } & NWG & $0.40 \pm 0.08$ & $0.46 \pm 0.08$ & $-4.878^{* *}$ & 2.807 & 1.222 & $15.508^{* * *}$ \\
\hline & BWG & $0.38 \pm 0.05$ & $0.38 \pm 0.05$ & -0.833 & & & \\
\hline & CG & $0.38 \pm 0.09$ & $0.33 \pm 0.08$ & 2.055 & & & \\
\hline \multicolumn{8}{|c|}{ Shoulder flexion strength (Nm) } \\
\hline \multirow[t]{3}{*}{ Dominance } & NWG & $26.86 \pm 3.97$ & $32.33 \pm 4.35$ & $-5.363^{* * *}$ & $3.491^{*}$ & 0.000 & $12.942^{* * *}$ \\
\hline & BWG & $33.35 \pm 9.01$ & $33.39 \pm 6.97$ & -0.032 & & & $a, b>c$ \\
\hline & CG & $29.03 \pm 5.80$ & $23.48 \pm 5.37$ & $2.716^{*}$ & & & \\
\hline \multirow[t]{3}{*}{ Nondominance } & NWG & $25.46 \pm 4.25$ & $31.17 \pm 6.91$ & $-3.975^{* *}$ & $4.096^{*}$ & 0.616 & $13.830^{* * *}$ \\
\hline & BWG & $30.34 \pm 6.80$ & $33.27 \pm 5.39$ & -1.751 & & & $a, b>c$ \\
\hline & CG & $28.13 \pm 5.88$ & $21.70 \pm 4.68$ & $3.754^{* *}$ & & & \\
\hline \multicolumn{8}{|c|}{ Shoulder extension strength (Nm) } \\
\hline \multirow[t]{3}{*}{ Dominance } & NWG & $34.53 \pm 3.64$ & $39.51 \pm 6.50$ & $-2.603^{*}$ & 2.789 & $8.652^{* *}$ & $4.145^{*}$ \\
\hline & BWG & $34.51 \pm 10.04$ & $39.08 \pm 11.90$ & $-3.730^{* *}$ & & & \\
\hline & CG & $30.05 \pm 4.43$ & $28.81 \pm 6.36$ & 0.726 & & & \\
\hline \multirow[t]{3}{*}{ Nondominance } & NWG & $32.13 \pm 3.76$ & $38.07 \pm 5.74$ & $-3.224^{*}$ & $6.420^{* *}$ & $8.514^{* *}$ & $9.887^{* *}$ \\
\hline & BWG & $30.45 \pm 10.99$ & $39.31 \pm 7.31$ & $-4.097^{* *}$ & & & $a, b>c$ \\
\hline & CG & $28.18 \pm 5.10$ & $24.00 \pm 2.79$ & 1.896 & & & \\
\hline
\end{tabular}

Values are present as mean \pm standard deviation.

NWG, Nordic walking group (a); BWG, brisk walking group (b); CG, control group (c).

${ }^{*} P<0.05$, ${ }^{* *} P<0.01$. ${ }^{* *} P<0.001$. 
On the other hand, no significant improvement was existed in BWG and CG.

\section{Comparison of shoulder flexion among groups after 8-week intervention}

In both DN and NDN shoulder, the strength of shoulder flexion was affected by main effect (Group) $(P<0.05)$, and significant interaction effect (Group $\times$ Time) $(P<0.001)$ was also found. After 8 -week intervention, NWG and BWG showed a significantly improved shoulder flexion compared to CG (Table 2). The strength of shoulder flexion in NWG was significantly enhanced in both $\mathrm{DN}(P<0.001)$ and NDN $(P<0.01)$ shoulder, however, no significant improvement was found in BWG. In CG, there was a noticeable decreased strength in both $\mathrm{DN}(P<0.05)$ and NDN $(P<0.01)$.

\section{Comparison of shoulder extension among groups after 8-week intervention}

DN shoulder was significantly affected by one main effect (Time) $(P<0.01)$, however, NDN shoulder was impacted by two main effects (Group and Time) $(P<0.01)$. In addition, both DN and NDN shoulder extension were significantly affected by the interaction effect (Group and Time) $(P<0.05$ and $P<0.01$, respectively). In NDN shoulder, NWG and BWG showed a significantly improved shoulder extension performance $(P<0.01)$ compared to CG, but not in DN shoulder (Table 2). After 8-week training, the strength of shoulder extension in both NWG $(P<0.05)$ and BWG $(P<0.01)$ was meaningfully improved in $\mathrm{DN}$ and NDN shoulder, however, no significant difference was found in CG.

\section{DISCUSSION}

The purpose of our study was to investigate whether the regular-basis NW training in healthy middle-aged woman have a beneficial effect to balance the asymmetric muscular strength between DN and NDN hand and shoulder. In our result, one primary finding was that 8 weeks of NW training program can improve the HS in NDN side, which ultimately ameliorates the discrepancy of muscular strength between the HS of DN and NDN.

In previous research, it was reported that brisk walking program did not induce a significant improvement of muscular strength in both DN and NDN side after the intervention. Another study reported that 12-week NW training effectively improved the strength of upper extremity in elderly women com- pared to normal walking group (Song et al., 2013). Moreover, when the 12 weeks of NW was applied to the elderly female subject diagnosed with osteopenia and osteoporosis, its training effect significantly enhances the HS of dominant hand. As our study showed that NW program can improve the HS in NDN side compared to other two groups, NW program may be a potential exercise intervention that provides the multiple kineological movement, enhancing not only the HS, but also cardio-pulmonary function by walking. Our study design could compare the effect NW on both DN and NDN muscular strength, however, other studies (Ossowski et al., 2016; Song et al., 2013) had a scientific limitation that examined only the strength of DN side. In this context, the effect of NW after the intervention may not be clearly investigated without the comparison of DN and NDN bodily area. As the characteristics of NW encourages the exerciser to move the whole upper extremity, it can be hypothesized that NW may enhance the strength of NDN shoulder flexion and extension as well as HS of NDN side. Accordingly, we showed that NW program can also increase the shoulder muscular strength of NDN side as well as that of DN, which potentiates the muscular balance between the DN and NDN shoulder. Since the participant used the pole creating the natural weight to upper extremity, it can mobilize more muscle activation through the action of continuous holding and pulling. In doing so, the strength of upper extremity in NDN could also obtain the kinesiological opportunity as the DN side is under the effect of NW training. In this sense, regular NW program can be an approachable exercise intervention that may prevent the potential decrease of muscular strength in NDN side of body.

The limitations of our study were that the strength of HS and shoulder were evaluated by only functional aspect. As the muscular strength can also be defined based on muscle size and thickness (Abe and Loenneke, 2015; Uthaikhup et al., 2015), the effect of $\mathrm{NW}$ on the change of muscular strength should be examined by the clinical approach such as ultrasound imaging technique. Moreover, the effect of NW training needs to be examined in the subject who undergoes a breast cancer operation known to cause the restricted mobility of shoulder joint, thereby verifying the NW as a rehabilitation method to balance the strength of bilateral muscle between DN and NDN shoulder.

In conclusion, our study demonstrated that regular-basis 8-week NW training can significantly improve the muscle strength of upper extremity in NDN side, and be more effective exercise intervention in enhancing the HS of NDN side than brisk walking method could. 


\section{CONFLICT OF INTEREST}

No potential conflict of interest relevant to this article was reported.

\section{REFERENCES}

Abe T, DeHoyos DV, Pollock ML, Garzarella L. Time course for strength and muscle thickness changes following upper and lower body resistance training in men and women. Eur J Appl Physiol 2000;81:174-180.

Abe T, Loenneke JP. Handgrip strength dominance is associated with difference in forearm muscle size. J Phys Ther Sci 2015;27:2147-2149.

Bagesteiro LB, Sainburg RL. Handedness: dominant arm advantages in control of limb dynamics. J Neurophysiol 2002;88:2408-2421.

Church TS, Earnest CP, Morss GM. Field testing of physiological responses associated with Nordic Walking. Res Q Exerc Sport 2002;73:296300 .

Figueiredo S, Finch L, Mai J, Ahmed S, Huang A, Mayo NE. Nordic walking for geriatric rehabilitation: a randomized pilot trial. Disabil Rehabil 2013;35:968-975.

Goble DJ, Brown SH. The biological and behavioral basis of upper limb asymmetries in sensorimotor performance. Neurosci Biobehav Rev 2008;32:598-610.

Jarjour N, Lathrop JA, Meller TE, Roberts KS, Sopczak JM, Van Genderen
$\mathrm{KJ}$, Moyers P. The 10\% rule: grip strength and hand dominance in a factory population. Work 1997;8:83-91.

Ossowski ZM, Skrobot W, Aschenbrenner P, Cesnaitiene VJ, Smaruj M. Effects of short-term Nordic walking training on sarcopenia-related parameters in women with low bone mass: a preliminary study. Clin Interv Aging 2016;11:1763-1771.

Petersen P, Petrick M, Connor H, Conklin D. Grip strength and hand dominance: challenging the 10\% rule. Am J Occup Ther 1989;43:444447.

Song MS, Yoo YK, Choi CH, Kim NC. Effects of nordic walking on body composition, muscle strength, and lipid profile in elderly women. Asian Nurs Res (Korean Soc Nurs Sci) 2013;7:1-7.

Takeshima N, Islam MM, Rogers ME, Rogers NL, Sengoku N, Koizumi D, Kitabayashi Y, Imai A, Naruse A. Effects of nordic walking compared to conventional walking and band-based resistance exercise on fitness in older adults. J Sports Sci Med 2013;12:422-430.

Uthaikhup S, Wannaprom N, Kummaung P. Effects of gender and hand dominance on size of the lower trapezius muscle. Muscle Nerve 2015; 52:576-579.

Virág A, Karóczi CK, Jakab Á, Vass Z, Kovács É, Gondos T. Short-term and long-term effects of nordic walking training on balance, functional mobility, muscle strength and aerobic endurance among Hungarian community-living older people: a feasibility study. J Sports Med Phys Fitness 2015;55:1285-1292. 